\section{El tratamiento postnatal con melatonina modula la expresión de agentes prostanoides en pulmón de neonatos de oveja con hipertensión pulmonar}

\author{
SIMÓN A. AGUILAR ${ }^{1, \mathrm{a}}$, PAMELA V. ARIAS ${ }^{1, \mathrm{a}}$, \\ IGNACIO CANQUIL ${ }^{1, a}$, GERMÁN EBENSPERGER ${ }^{1, b, e}$, \\ ANÍBAL J. LLANOS ${ }^{1,2}$, ROBERTO V. REYES ${ }^{1, b, e}$, \\ ALEJANDRO GONZÁLEZ-CANDIA ${ }^{1, \mathrm{c}, \mathrm{f}}$, EMILIO A. HERRERA $^{1,2, \mathrm{~d}, \mathrm{e}}$
}

\section{Melatonin modulates the expression of pulmonary prostanoids}

Background: Living above 2,500 meters in hypobaric conditions induces pulmonary arterial hypertension of the neonate (PAHN), a syndrome whose main features are: pathological remodeling of the pulmonary vessels, abnormal vascular reactivity and increased oxidative stress. Melatonin could have pulmonary antioxidant, anti-remodeling and vasodilating properties for this condition. Aim: To determine the effect of melatonin at the transcript level of prostanoid pathways in the lung of neonatal lambs gestated and born under hypobaric hypoxia. Material and Methods: Vehicle (1.4\% of ethanol, $n=6)$ or melatonin $\left(1 \mathrm{mg}^{*} \mathrm{~kg}^{-1}, n=5\right)$ were administered from the postnatal day 4 to 21 to lambs gestated and born at 3,600 meters above sea level. After one week of treatment completion, lung tissue was obtained, the transcript and protein levels of prostanoid synthases and receptors were assessed by RT-PCR and Western Blot. Results: Melatonin induced the expression of prostacyclin synthase transcript and increased protein expression of the prostacyclin receptor. In addition, the treatment decreased the expression of transcript and protein of cyclooxygenase-2, without changes in the expression of the prostanoid vasoconstrictor (thromboxane) pathway. Conclusions: Postnatal treatment with melatonin increases the expression of the prostacyclin-vasodilator pathway without changing the vasoconstrictor thromboxane pathway. Further, the decreased COX-2 induced by melatonin could be an index of lesser oxidative stress and inflammation in the lung.

(Rev Med Chile 2019; 147: 281-288)

Key words: Hypoxia; Hypertension, Pulmonary; Newborn; Melatonin; Prostaglandins.

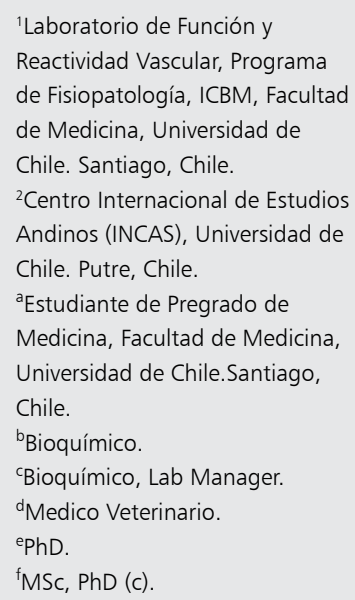

Fuente de Financiamiento: Proyecto Fondecyt 1151119. Los autores declaran no tener conflictos de interés.

Recibido el 1 de octubre de 2018 , aceptado el 1 de abril de 2019 .

Correspondencia a:

Emilio A. Herrera

Av. Salvador 486, Providencia.

Santiago, Chile.

eherrera@med.uchile.cl

Alejandro González-Candia Av. Salvador 486, Providencia. Santiago, Chile.

a.gonzalez@ciq.uchile.cl

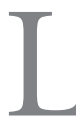

a hipertensión arterial pulmonar neonatal (HAPN) es una condición fisiopatológica definida como un aumento de la presión arterial pulmonar media sobre $25 \mathrm{mmHg}$, asociada a algún grado de hipoxemia como resultado de una inadecuada vasodilatación pulmonar al nacimiento ${ }^{1,2}$. Esta condición clínica tiene una prevalencia de hasta 7/1.000 nacidos vivos en tierras bajas, representando un grave problema clínico con alta morbimortalidad ${ }^{3}$. Sin embargo, su prevalencia es desconocida en alturas geográficas superiores a 2.500 metros sobre el nivel del mar (msnm), aunque se ha sugerido que podría alcanzar hasta $10 \%$ en recién nacidos vivos ${ }^{4}$. 
La patogénesis de la HAPN se caracteriza por una disfunción endotelial, estrés oxidativo aumentado, aumento de parámetros inflamatorios y remodelamiento vascular patológico determinado por una hiperplasia e hipertrofia de la capa media, engrosamiento de la adventicia e íntima y un aumento de la matriz extracelular, provocando una vasorreactividad anormal tendiente a un tono vasoconstrictor $^{5-7}$.

Entre los factores involucrados en la vasorreactividad pulmonar, destacan la vía dependiente de óxido nítrico $(\mathrm{NO})$ y la vía prostanoide, las cuales tienen su acción en la musculatura lisa vascular pulmonar ${ }^{5,6,8}$. Los prostanoides se generan a partir de ácido araquidónico presente en membranas celulares, el cual es metabolizado por las enzimas ciclooxigenasa-1 (COX-1, isoforma constitutiva) o ciclooxigenasa-2 (COX-2, isoforma inducible), que sintetizan prostaglandina $\mathrm{PGH}_{2}^{8,9}$. A partir de $\mathrm{PGH}_{2}$ y la acción de sintasas específicas, se generan las prostaglandinas $\mathrm{PGD}_{2}, \mathrm{PGE}_{2}, \mathrm{PGF}_{2 \alpha}$, prostaciclina $\left(\mathrm{PG}_{12}\right)$ y tromboxano $(\mathrm{TX})$, cada una asociada a un receptor de membrana específico DP1, Ep1-4, FP , Ip y Tp, respectivamente ${ }^{9,10}$.

Los agentes prostanoides se pueden clasificar de acuerdo con su acción vasoactiva. En el primer grupo se encuentran las prostaglandinas vasodilatadoras $\mathrm{PGD}_{2}$ y $\mathrm{PGI}_{2}$, catalizadas por la prostaglandina $\mathrm{D}_{2}$ sintasa $\left(\mathrm{PGD}_{2 \mathrm{~S}}\right)$ y $\mathrm{PGI}$ sintasa $\left(\mathrm{PGI}_{2 \mathrm{~S}}\right)$, respectivamente. Los prostanoides vasoconstrictores son $\mathrm{PGF}_{2}$ producto de la prostaglandina $\mathrm{F}$ sintasa (PG$\mathrm{F}_{2 \mathrm{~S}}$ ), TX formado por tromboxano sintasa (TXs) y $\mathrm{PGE}_{2}$ generado por la prostaglandina $\mathrm{E}$ sintasa $\left(\mathrm{PGE}_{2 \mathrm{~S}}\right)$. Los prostanoides vasodilatadores activan a la proteína Gs, actuando sobre la enzima adenilato ciclasa, generando AMPc, molécula que activa la proteína quinasa $\mathrm{A}$ (PKA), lo que finalmente disminuye la biodisponibilidad de $\mathrm{Ca}^{2+}$ intracelular asociado a una vasodilatación arterial. Por otro lado, los prostanoides vasoconstrictores pueden activar dos tipos diferentes de proteína G. Los receptores $\mathrm{Tp}$, Ep1 y FP activan a la proteína Gq que permite la formación de IP3, movilizando $\mathrm{Ca}^{2+}$ desde el retículo sarcoplasmático al citosol, generando una activación de la maquinaria contráctil celular. En contraste, el receptor Ep3 activa la proteína $\mathrm{Gi}$, que aumenta los niveles de $\mathrm{Ca}^{2+}$ intracelulares al inhibir la acción de la adenilato ciclasa ${ }^{9,10}$.

Se ha descrito un rol importante de las especies reactivas de oxígeno (ERO) en el aumento de la reactividad vasoconstrictora pulmonar asociado a $\mathrm{HTP}^{11,12}$. Asimismo, las ERO pueden unirse directamente al NO disminuyendo su biodisponibilidad ${ }^{5,13}$. Finalmente, las ERO afectarán la relajación normal de la arteria pulmonar en respuesta a vasodilatadores y favorecen el remodelamiento patológico ${ }^{13}$.

Actualmente existe solo un tratamiento aprobado por la Food \& Drug Administration de Estados Unidos de Norteamérica para la HAPN, que consiste en la administración de NO inhalado, que induce una rápida y selectiva vasodilatación pulmonar ${ }^{1,14}$. Sin embargo, ensayos clínicos revelan que cerca de $40 \%$ de los pacientes no responden o responden parcialmente a esta terapia ${ }^{1,2}$, por ello, nuevos tratamientos son necesarios para combatir esta condición. Por esta razón, en nuestra búsqueda de nuevos tratamientos para HAPN, hemos postulado a melatonina, que tiene propiedades vasodilatadoras, antioxidantes y antiremodelante en la circulación pulmonar neonatal ${ }^{15,16}$. Estudios muestran diversos efectos de melatonina a nivel vascular, como mejorar la capacidad vasodilatadora y función endotelial pulmonar ${ }^{15}$, ser agente antirremodelante previniendo la excesiva proliferación de la túnica media ${ }^{16,17}$ y antioxidante, pudiendo neutralizar directamente a las ERO (scavenger $)^{18}$. Además, se ha demostrado que melatonina induce la expresión del transcrito y/o proteínas de enzimas antioxidantes como la superóxido dismutasa y la catalasa ${ }^{15}$, y también modula negativamente los mecanismos prooxidantes como la eNOS desacoplada y las lipooxigenasas ${ }^{18}$. Asimismo, se han descrito escasos efectos adversos exclusivamente durante la gestación ${ }^{19,20}$. Sin embargo, aún se desconocen las vías vasodilatadoras pulmonares que son favorecidos por melatonina.

En este estudio se evaluó el efecto de la administración neonatal de melatonina sobre las vías prostanoides pulmonares. Específicamente, se analizaron los efectos de melatonina en el nivel de transcrito y de proteína de sintasas y receptores de la vía prostanoide vasoconstrictora y vasodilatadora en tejido pulmonar de neonatos de ovejas con HAPN gestados, nacidos y criados en hipoxia crónica.

\section{Material y Método}

Todos los procedimientos experimentales fueron aprobados por el Comité de Bioética sobre Investigación en Animales de la Facultad de 
Medicina de la Universidad de Chile (CBA\#0761), además de seguir las indicaciones de las guías internacionales de cuidado y uso de animales en investigación y el reporte de sus resultados ${ }^{21}$.

\section{Muestras biológicas}

Para este estudio se utilizaron 10 corderos (Ovis aries) recién nacidos con hipertensión pulmonar, modelo caracterizado en estudios previos ${ }^{7,15,16,22,23}$. Las ovejas madres de estos corderos concibieron y gestaron en hipoxia hipobárica en el Centro Internacional de Estudios Andinos de la Universidad de Chile (INCAS, Putre a $3.600 \mathrm{msnm}$ ). Al nacer, los corderos fueron estudiados y mantenidos con sus madres en el mismo centro de investigación. Los grupos experimentales se dividieron en: grupo control (C), tratados con vehículo $\left(0,5 \mathrm{~mL}^{\star} \mathrm{kg}^{-1}\right.$ de etanol al $1,4 \%, \mathrm{n}=5$ ) y grupo tratado $(\mathrm{M})$ con melatonina (Sigma-Aldrich código M5259, Merck KGaA, Darmstadt, Alemania; $1 \mathrm{mg}^{\star} \mathrm{kg}^{-1}$ de melatonina en $1,4 \%$ etanol, $n=5$ ). Los tratamientos fueron administrados diariamente, vía oral, a las $20 \mathrm{~h}$, desde el día 4 al 21 postnatal. Para evaluar que los cambios fisiológicos atribuidos a melatonina perduraran en el tiempo, los corderos se mantuvieron por 7 días sin tratamiento (desde el día 22 al 28 postnatal). Finalmente, los animales fueron eutanasiados (tiopental sódico, Opet, Laboratorios PRO-VET, Santiago, Chile; 100 $\mathrm{mg}^{\star} \mathrm{kg}^{-1}$, vía endovenosa) a los 28 días de edad y se obtuvieron muestras de parénquima pulmonar, que fueron congeladas y mantenidas en nitrógeno líquido $\left(-197^{\circ} \mathrm{C}\right)$ hasta su utilización.

\section{Expresión de mRNA de sintasas y receptores de la vía prostanoide}

La purificación de RNA en tejido pulmonar, síntesis de ADN complementario (cDNA) y la amplificación mediante reacción en cadena de la polimerasa con transcriptasa inversa (RT-PCR) se efectuó como se ha descrito previamente ${ }^{15}$. Para esto, se diseñaron partidores para la amplificación de los genes de ciclooxigenasas, agentes prostanoides vasoconstrictores y vasodilatadores (Tabla 1).

Tabla 1. Partidores para la amplificación de los genes

\begin{tabular}{|c|c|c|c|c|}
\hline Partidor & Secuencia 5'-3' & $\operatorname{Tm}\left({ }^{\circ} \mathrm{C}\right)$ & Ciclos & $\begin{array}{l}\text { Tamaño del } \\
\text { amplicon (pb) }\end{array}$ \\
\hline $\begin{array}{c}\text { COX } 1 \\
F \\
R\end{array}$ & $\begin{array}{l}\text { CTCATCGGGGAGACCATCAA } \\
\text { AGAAAAGGCGTCCACCAGG }\end{array}$ & 62,5 & 35 & 276 \\
\hline $\begin{array}{c}\text { COX } 2 \\
F \\
R\end{array}$ & $\begin{array}{l}\text { CAGAATGGGGCGATGAGC } \\
\text { GATGCCAGTGGTAGAGCGTG }\end{array}$ & 62 & 35 & 204 \\
\hline $\begin{array}{c}\mathrm{TX}_{\mathrm{S}} \\
\mathrm{F} \\
\mathrm{R}\end{array}$ & $\begin{array}{l}\text { CGGATTTTGGCCCAATAAGA } \\
\text { AAAGACCTGACGGACGATG }\end{array}$ & 59 & 33 & 192 \\
\hline $\begin{array}{c}\mathrm{TP}_{2} \\
\mathrm{~F} \\
\mathrm{R}\end{array}$ & $\begin{array}{l}\text { GCGAGGTGGAGATGATGGT } \\
\text { GCTCAGGCGAGGGTAGAAG }\end{array}$ & 62 & 33 & 257 \\
\hline $\begin{array}{c}\mathrm{PGI}_{2 \mathrm{~S}} \\
\mathrm{~F} \\
\mathrm{R}\end{array}$ & $\begin{array}{l}\text { GGCTGGAGAGTTACCTGCTG } \\
\text { ATCTGTGAAATGGGCTGCTC }\end{array}$ & 60 & 35 & 217 \\
\hline $\begin{array}{c}\text { IP } \\
F \\
R\end{array}$ & $\begin{array}{l}\text { GGTTGACCACCTGATTCTGC } \\
\text { ACGGACTTTCGGAAGAGGAT }\end{array}$ & 60 & 33 & 192 \\
\hline $\begin{array}{c}18 \mathrm{~s} \\
\mathrm{~F} \\
\mathrm{R}\end{array}$ & $\begin{array}{l}\text { GTAACCCGTTGAACCCCATT } \\
\text { CCATCCAATCGGTAGTAGCG }\end{array}$ & 58 & 20 & 152 \\
\hline
\end{tabular}

Abreviaturas: COX-1: ciclooxigenasa 1; COX-2 : ciclooxigenasa 2; $\mathrm{TX}_{\mathrm{s}}$ : tromboxano sintasa; $\mathrm{TP}_{2}$ : receptor de tromboxano; $\mathrm{PGI}_{25}$ : rostaciclina sintasa; Ip: receptor de prostaciclina; F: partidor forward; R: partidor reverse. 
Los productos de PCR fueron separados por electroforesis en geles de agarosa $2 \%$ en buffer de tris, acetato y ácido etilendiaminotetraacético (Thermo Fischer Scientific, Waltham, MA, USA), y luego se visualizaron con luz ultravioleta y fotografiaron digitalmente. Finalmente, la imágenes se cuantificaron mediante densitometría con un programa computacional (Scion Image Beta 4.02 Win; Scion Corporation, MD, USA).

\section{Expresión de proteina de sintasas y receptores de la vía prostanoides}

La extracción de proteína a partir de pulmón se realizó mediante homogenización mecánica en buffer RIPA como se describe en trabajos de Torres F et al y Astorga CA et al ${ }^{15,16}$. Los lisados de proteína (1-40 $\mu$ g según proteína) fueron separados por electroforesis en geles de poliacrilamida SDS-PAGE ( $4 \%$ de gel concentrador y $10 \%$ de gel de corrida al 10\%). La electroforesis se realizó en una cámara (Mini-PROTEAN Tetra Cell/ Blotting System, Bio-Rad, CA, USA), a un amperaje constante de $50 \mathrm{~mA}$ por $2 \mathrm{~h}$ aproximadamente. Posteriormente las proteínas fueron transferidas a una membrana de nitrocelulosa a $50 \mathrm{mV}$ por $2 \mathrm{~h}$. Finalmente, la expresión proteica de COX-1, COX-2, TXs, Tp, PGIs, Ip y $\beta$-actina se realizó por immunoblot utilizando anticuerpos específicos: anti-COX-1, 160108; anti-COX-2, 160116; anti-Txs, 160715; anti-Tp, 101882; anti PGIs, 160640 (Cayman Chemical, Ann Arbor, MI, USA); anti-Ip (Sc-365268, Santa Cruz Biotechnology Inc.) y anti $\beta$-actina (AC-15, Thermo Fisher Scientific, Waltham, MA, USA). La inmunodetección se realizó a través de anticuerpos secundarios conjugados con horseradish-peroxidase (HRP) (Thermo Fisher Scientific, Waltham, MA, USA), anti-conejo o ratón, según corresponda. Posteriormente, la membrana fue montada en una cámara reveladora C-Digit Modelo 3600 (LI-COR Biosciences, Lincoln, NE, USA), y se cuantificó la expresión proteica por densitometría clásica utilizando el programa Image Studio 3.1 Imagening Software (LI-COR Biosciences. Lincoln, NE, USA). Las densidades de las señales fueron expresadas como razón de $\beta$-actina para normalizar por carga.

\section{Análisis de datos \\ Los resultados se expresaron como promedio \pm error estándar de la media. Se determinó la}

distribución de la muestra mediante la prueba de Kolmogorov-Smirnov. La comparación entre grupos se realizó por prueba de Mann Whitney (prueba no paramétrica del t test) y las diferencias se consideraron significativas cuando $\mathrm{p} \leq 0,05$ (Prism 5.0; GraphPad Software, San Diego, CA, USA) $)^{15,16}$.

\section{Resultados}

\section{Expresión de mRNA y proteína de ciclooxigenasas}

La expresión de transcrito (mRNA) para COX-1 fue similar entre grupos (Figura 1A,C). En contraste, melatonina indujo una disminución significativa en la expresión de mRNA de COX2 en comparación con el grupo control (Figura 1B,C). Con respecto a la expresión de proteínas de las ciclooxigenasas, no hubo cambios en los niveles de COX-1 entre los grupos analizados (Figura 1D, F). Sin embargo, el tratamiento con melatonina logró disminuir la expresión proteica de COX-2 (Figura 1E,F) en comparación con el grupo control.

\section{Expresión de mRNA y proteína de la vía vasoconstrictora prostanoide}

Los niveles de expresión de la sintasa de tromboxano (TXs) a nivel de transcrito (Figura $2 \mathrm{~A}, \mathrm{C}$ ) y de proteína (Figura 2 D, F) no mostraron diferencias significativas entre los grupos analizados. A la vez, tampoco se observaron diferencias entre grupos en la expresión proteica (Figura 2 E, F) o nivel de transcrito (Figura $2 \mathrm{~B}, \mathrm{C}$ ) del receptor de tromboxano $(\mathrm{Tp})$.

\section{Expresión de mRNA y proteína de la vía vasodilatadora prostanoide}

El tratamiento con melatonina indujo un aumento significativo en la expresión de transcrito de la prostaciclina sintasa ( $\mathrm{PGI}_{2} \mathrm{~s}$ ) en comparación al grupo control (Figura $3 \mathrm{~A}, \mathrm{C}$ ), aunque a nivel de su receptor Ip no se observaron diferencias significativas a nivel de transcrito (Figura $3 \mathrm{~B}$, C). En contraste, no se observaron cambios en la expresión proteica de la sintasa de prostaglandina $\mathrm{PGI}_{2}$ (Figura $3 \mathrm{D}, \mathrm{F}$ ), mientras que el tratamiento con melatonina aumentó de manera significativa la expresión proteica del receptor Ip (Figura 3 E, F) en comparación con el grupo control. 
A.

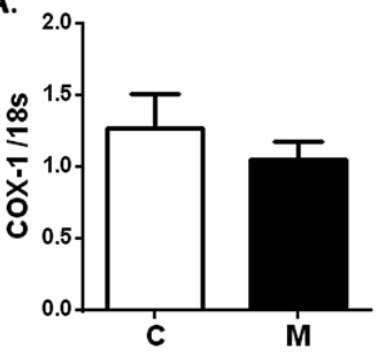

D.

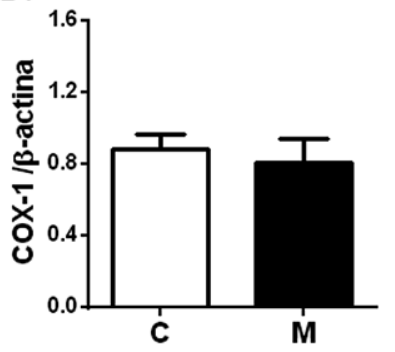

B.

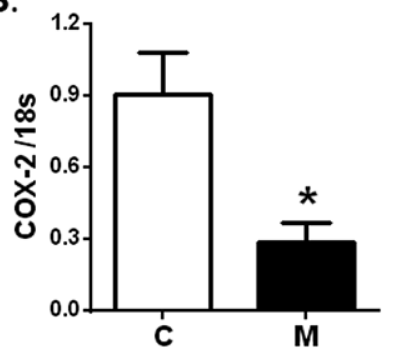

E.

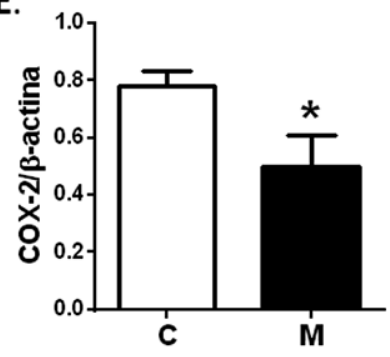

C.

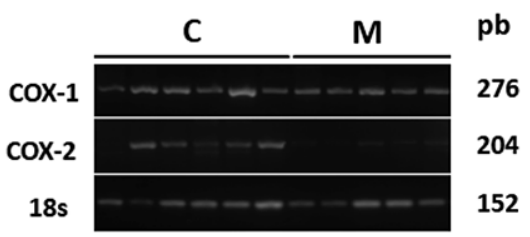

F.

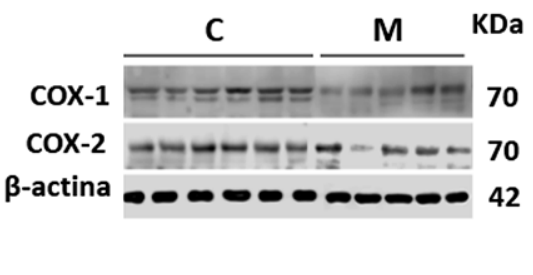

Figura 1. Niveles de transcrito y proteína de ciclooxigenasas. Los resultados se expresan como promedio \pm E.S.M. de mRNA para COX-1 (A) y COX-2 (B) y proteína para COX-1 (D) y COX-2 (E). Se muestran imágenes representativas de los ensayos de RT-PCR (C) y Western blot (F). Los grupos son control $(C, n=6$, barras blancas) y tratados con melatonina $(M, n=5$, barras negras). Diferencias significativas $(p \leq 0,05)={ }^{*}$ vs $C$.

A.

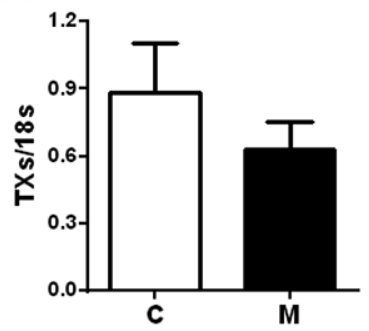

D.

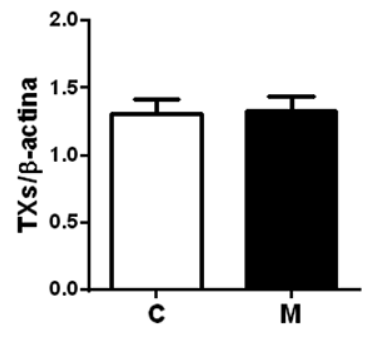

B.

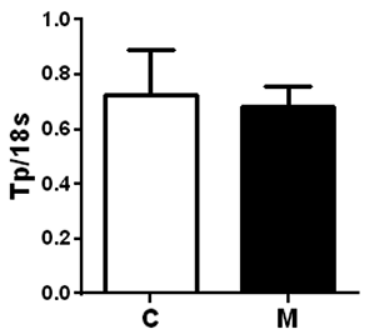

E.

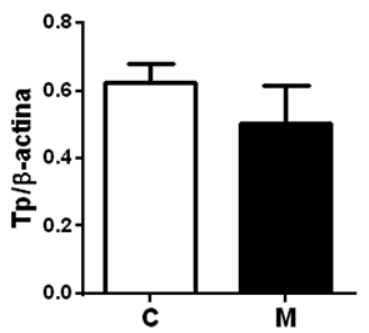

C.

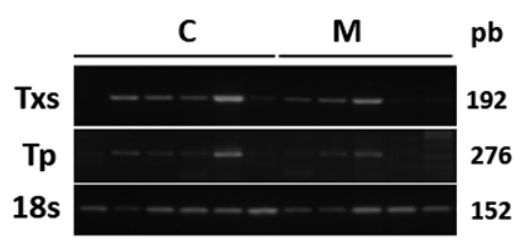

F.

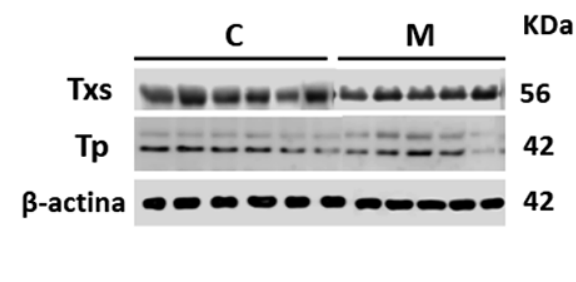

Figura 2. Niveles de expresión de la sintasa y receptor de tromboxano. Los resultados se expresan como promedio \pm E.S.M. de mRNA para TXs (A), Tp (B) y proteína de TXs (D) y Tp (E). Se muestran imágenes representativas de los ensayos de RT-PCR (C) y Western blot (F). Los grupos son control (,$n=6$, barras blancas) y tratados con melatonina ( $M, n=5$, barras negras). Diferencias significativas $(p \leq 0,05)={ }^{*}$ vs $C$. 
A.

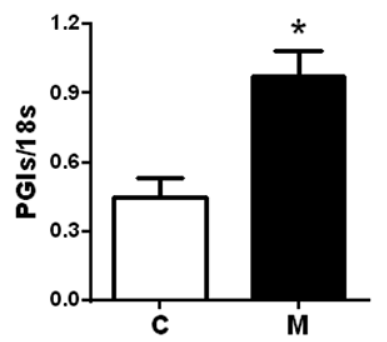

D.

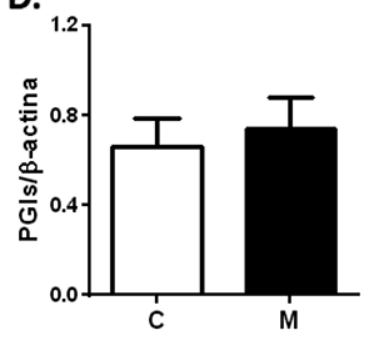

B.

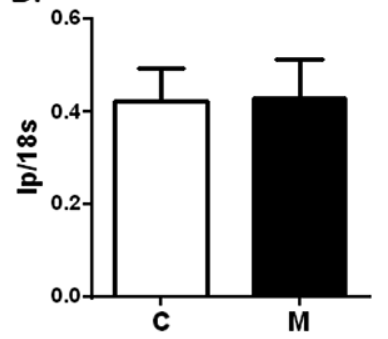

E.

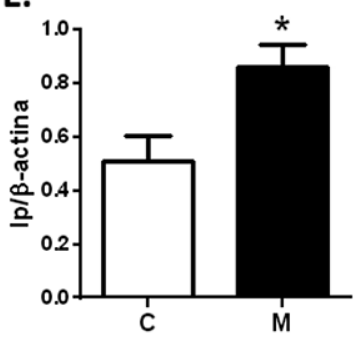

C.

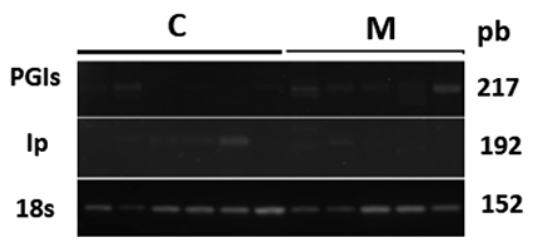

F.

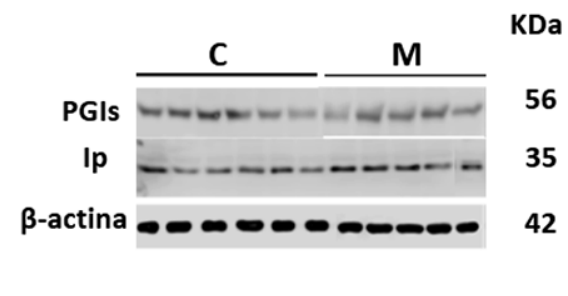

Figura 3. Niveles de expresión de la sintasa y receptor de prostaciclina. Los resultados se expresan como promedio \pm E.S.M. de mRNA para PGIs (A), Ip (B) y proteína de PGIs (D) y Ip (E). Se muestran imágenes representativas de los ensayos de RT-PCR (C) y Western blot (F). Los grupos son control $(C, n=6$, barras blancas) y tratados con melatonina ( $M, n=5$, barras negras). Diferencias significativas $(p \leq 0,05)={ }^{*}$ vs $C$.

\section{Discusión}

Los resultados de este estudio demuestran que un tratamiento con melatonina durante las primeras tres semanas de vida con una dosificación farmacológica diaria de $1 \mathrm{mg}^{\star} \mathrm{Kg}^{-1}$ logró modular de manera diferencial la expresión de las vías prostanoides en pulmón de corderos. En primer lugar, melatonina logró disminuir a nivel de transcrito y de proteína la COX-2 sin cambios en la expresión de COX-1. Se ha demostrado que la expresión de COX-2 (forma inducible de las ciclooxigenasas) aumenta en diferentes condiciones fisiopatológicas, como estrés oxidativo, cuadros inflamatorios y proliferación celular asociada a crecimiento tumoral en diferentes tejidos ${ }^{8,24,25}$. Por lo tanto, una disminución de la COX-2 podría ser indicador de menor estrés oxidativo e inflamación pulmonar. Con respecto a lo anterior, se ha demostrado que melatonina disminuye el estrés oxidativo y la presión pulmonar en neonatos ${ }^{15,16}$ $y$ adultos $^{26}$ de modelos animales. Los efectos antioxidantes de melatonina están asociados a sus características de: 1) scavenger de radicales libres, 2) inducción de la maquinaria enzimática antioxidante y 3 ) disminución de la expresión de enzimas prooxidantes ${ }^{27}$, lo cual disminuye el tono oxidativo en el territorio pulmonar ${ }^{15,17,18}$. Además, melatonina puede modular la producción de diferentes mediadores inflamatorios como TNF- $\alpha$ vía receptor, disminuyendo de esta forma el tono inflamatorio a nivel celular y la sobreexpresión de COX-2 $28-30$. Por otro lado, se ha visto que la ausencia de COX-2 trae como consecuencia una exacerbación de la hipertensión pulmonar y del remodelamiento patológico ${ }^{31}$, sin embargo, estos estudios solo modifican la expresión genética de COX-2, sin modificar otros parámetros involucrados en la vasorreactividad.

Se ha demostrado que la administración de melatonina normaliza la sobreexpresión de COX2, TNF- $\alpha$ e iNOS disminuyendo, además, los niveles de NF- $\kappa \mathrm{B}$ en células cultivadas y modelos animales $^{30}$. En este sentido es relevante destacar que la acción de melatonina tiende a normalizar los parámetros inflamatorios y permitir una expresión a un nivel similar al fisiológico de COX-2.

En términos del balance vasoactivo que pre- 
senta el lecho pulmonar, el cuadro clínico de la HAPN se caracteriza por presentar un marcado aumento de los vasoconstrictores a nivel pulmonar ${ }^{13,14}$. Si bien este trabajo no mostró diferencias en la expresión a nivel de mensajero ni de proteína de la vía dependiente de tromboxano (TXs-Tp), sí mostró un aumento del transcrito en la expresión de sintasa PGI, la cual favorece un balance prostanoide vasodilatador ${ }^{24,25}$. Interesantemente, además, melatonina indujo un aumento de la expresión del receptor Ip, favoreciendo la acción vasodilatadora de $\mathrm{PGI}_{2}$.

La hipertensión pulmonar posee un componente inflamatorio ${ }^{25}$, y en base a esto, se ha demostrado que melatonina es capaz de inhibir las traslocación y acción transcripcional del NFא-B ${ }^{29,32}$ que, a su vez, activa el factor TNF- $\alpha$, suprimiendo la expresión del transcrito de PGIs ${ }^{32}$. Asimismo, la acción antioxidante y antiinflamatoria de melatonina estaría inhibiendo la traslocación al núcleo de NFא-B, impidiendo la activación de TNF- $\alpha$, lo que conduciría a un aumento en los niveles de transcrito de PGIs. A nivel proteico, melatonina logró inducir la expresión del receptor Ip sin cambiar la sintasa de prostaciclina PGIs, lo que sugiere un aumento en la sensibilidad de la respuesta vasodilatadora de $\mathrm{PGI}_{2}$. La actividad de PGIs y el efecto de $\mathrm{PGI}_{2}$ debiese ser foco de estudio en futuras investigaciones para confirmar esta sugerencia.

La HPTN se asocia a una alteración de la respuesta vascular pulmonar, comandada principalmente por el endotelio y las células musculares lisas. Si bien este trabajo solo estudió el efecto de melatonina en la vasorreactividad mediada por prostanoides, esta vía junto a la oxidonitrérgica son las principales responsables de mantener el tono a nivel vascular. Estudios previos han demostrado que una administración aguda de melatonina logra aumentar ex vivo la vasodilatación dependiente de óxido nítrico, además de inducir una respuesta vasodilatadora mediada por alguna vía independiente de óxido nítrico en arterias de resistencia pulmonar ${ }^{15}$.

Existe un amplio rango de dosis de melatonina (0,0005 - $100 \mathrm{mg}$ totales) descrito en la literatura, en los cuales no se han visto efectos adversos. En este sentido, este trabajo continúa la exploración de mecanismos vasoactivos realizada por trabajos anteriores ${ }^{15,16}$, los que mostraron efectos del tratamiento mejorando el estrés oxidativo y la función vascular.
Se concluye que el tratamiento postnatal con melatonina oral induce efectivamente la expresión de agentes prostanoides vasodilatadores, lo que ayudaría a contrarrestar la disfunción endotelial caracterizada por un predominio del tono vasoconstrictor propio del cuadro clínico de la HAPN.

De este estudio se infiere que el uso de melatonina tiene potenciales usos terapéuticos en la HAPN. Los efectos a nivel pulmonar persisten en el tiempo, a pesar del término de la administración del tratamiento. Por ello, consideramos que melatonina podría ser una potencial terapia coadyuvante para el tratamiento de esta patología.

Agradecimientos: Nuestros agradecimientos para los Srs. Carlos Brito, Gabino Llusco y René Vergara por la excelente asistencia técnica.

\section{Referencias}

1. Jain A, McNamara PJ. Persistent pulmonary hypertension of the newborn: Advances in diagnosis and treatment. Semin Fetal Neonatal Med 2015; 20 (4): 262-71.

2. Walsh-Sukys MC, Tyson JE, Wright LL, Bauer CR, Korones SB, Stevenson DK, et al. Persistent pulmonary hypertension of the newborn in the era before nitric oxide: practice variation and outcomes. Pediatr 2000; 105: 14-20.

3. Keyes LE, Armaza JF, Niermeyer S, Vargas E, Young DA, Moore LG. Intrauterine growth restriction, preeclampsia, and intrauterine mortality at high altitude in Bolivia. Pediatr Res 2003; 54 (1): 20-5.

4. Peñaloza D, Arias-Stella J. The heart and pulmonary circulation at high altitudes healthy highlanders and chronic mountain sickness. Circulation 2007; 115 (9): 1132-46.

5. Tabima D, Frizzell S, Gladwin M. Reactive Oxygen and Nitrogen Species in Pulmonary Hypertension. Free Radic Biol Med 2012; 52 (9): 1970-86.

6. Stenmark KR, Frid MG, Graham BB, Tuder RM. Dyna$\mathrm{mic}$ and diverse changes in the functional properties of vascular smooth muscle cells in pulmonary hypertension. Cardiovasc Res 2018; 114(4): 551-564.

7. Herrera EA, Ebensperger G, Krause BJ, Riquelme RA, Reyes RV, Capetillo M, et al. Sildenafil reverses hypoxic pulmonary hypertension in highland and lowland newborn sheep. Pediatr Res 2008; 63 (2): 169-75.

8. Smith WL, DeWitt DL, Garavito RM. Cyclooxygenases: Structural, cellular, and molecular biology. Annu Rev Biochem 2000; 69: 145-82. 
9. Narumiya S, Sugimoto Y, Ushikubi F. Prostanoid Receptors: Structures, Properties, and Functions. Physiol Rev 1999; 79 (4): 1193-226.

10. Bos CL, Richel DJ, Ritsema T, Peppelenbosch MP, Versteeg HH. Prostanoids and prostanoid receptors in signal transduction. Int J Biochem Cell Biol 2004; 36 (7): 1187-205.

11. Jernigan NL, Naik JS, Weise-Cross L, Detweiler ND, Herbert LM, Yellowhair TR, et al. Contribution of reactive oxygen species to the pathogenesis of pulmonary arterial hypertension. PLoS ONE 2017; 12 (6): e0180455.

12. Jaitovich A, Jourd'heuil D. A brief overview of nitric oxide and reactive oxygen species signaling in hypoxia-induced pulmonary hypertension. Adv Exp Med Biol 2017; 967: 71-81.

13. Wedgwood S, Steinhorn RH. Role of Reactive Oxygen Species in Neonatal Pulmonary Vascular Disease. Antioxid Redox Signal 2014; 21 (13): 1926-42.

14. Nair J, Lakshminrusimha S. Update on PPHN: mechanisms and treatment. Semin Perinatol 2014; 38 (2): 78-91.

15. Torres F, González-Candia A, Montt C, Ebensperger G, Chubretovic M, Serón-Ferré M, et al. Melatonin reduces oxidative stress and improves vascular function in pulmonary hypertensive newborn sheep. J Pineal Res 2015; 58 (3): 362-73.

16. Astorga CA, González-Candia A, Candia AA, Figueroa EG, Cañas D, Ebensperger G, et al. Melatonin decreases pulmonary vascular remodeling and oxygen sensitivity in pulmonary hypertensive newborn lambs. Front Physiol 2018; 9: 185.

17. Galano A, Tan D, Reiter RJ. Melatonin as a natural ally against oxidative stress: a physicochemical examination. J Pineal Res 2011; 51: 1-16.

18. Hardeland R. Antioxidative protection by melatonin: multiplicity of mechanisms from radical detoxification to radical avoidance. Endocrine 2005; 27 (2): 119-30.

19. González-Candia A, Veliz M, Araya C, Quezada S, Ebensperger G, Serón-Ferré M, et al. Potential adverse effects of antenatal melatonin as a treatment for intrauterine growth restriction: findings in pregnant sheep. Am J Obstet Gynecol 2016; 215 (2): 245.e1-7.

20. Renshall LJ, Morgan HL, Moens H, Cansfield D, Finn-Sell SL, Tropea T, et al. Melatonin Increases Fetal Weight in Wild-Type Mice but Not in Mouse Models of Fetal Growth Restriction. Front Physiol 2018; 9: 1141.

21. Grundy D. Principles and standards for reporting animal experiments in The Journal of Physiology and Ex- perimental Physiology. J Physiol 2015; 593 (12): 2547-9.

22. Herrera EA, Macchiavello R, Montt C, Ebensperger G, Díaz M, Ramírez S, et al. Melatonin improves cerebrovascular function and decreases oxidative stress in chronically hypoxic lambs. J Pineal Res 2014; 57 (1): $33-42$.

23. Herrera EA, Pulgar VM, Riquelme RA, Sanhueza EM, Reyes VR, Ebensperger G, et al. High altitude chronic hypoxia during gestation and after birth modifies cardiovascular responses in newborn sheep. Am J Physiol Regul Integr Comp Physiol 2007; 292 (6): 2234-40.

24. Smyth EM, Grosser T, Wang M, Yu Y, Fitzgerald GA. Prostanoids in health and disease. J Lipid Res 2009; 50 (Suppl): S423-S428.

25. Price LC, Wort SJ, Perros F, Dorfmüller P, Huertas A, Montani D, et al. Inflammation in pulmonary arterial hypertension. Chest 2012; 141 (1): 210-21.

26. Hung MW, Yeung HM, Lau CF, Poon AMS, Tipoe GL, Fung ML. Melatonin attenuates pulmonary hypertension in chronically hypoxic rats. Int J Mol Sci 2017; 18 (6): E1125.

27. González-Candia A, Veliz M, Carrasco-Pozo C, Castillo RL, Cárdenas JC, Ebensperger G, et al. Antenatal melatonin modulates an enhanced antioxidant/pro-oxidant ratio in pulmonary hypertensive newborn sheep. Redox Biol 2019; 22: 101128.

28. Favero G, Franceschetti L, Bonomini F, Rodella LF, Rezzani R. Melatonin as an Anti-Inflammatory Agent Modulating Inflammasome Activation. Int J Endocrinol 2017; 2017: 1835195.

29. Wang J, Xiao X, Zhang Y, Shi D, Chen W, Fu L, et al. Simultaneous modulation of COX-2, p300, Akt, and Apaf-1 signaling by melatonin to inhibit proliferation and induce apoptosis in breast cancer cells. J Pineal Res 2012; 53 (1): 77-90.

30. Tripathi DN, Jena GB. Effect of melatonin on the expression of Nrf2 and NF-kappaB during cyclophosphamide-induced urinary bladder injury in rat. J Pineal Res 2010; 48 (4): 324-31.

31. Fredenburgh LE, Liang OD, Macias AA, Polte TR, Liu $\mathrm{X}$, Riascos DF, Perrella MA. Absence of COX-2 exacerbates hypoxia-induced pulmonary hypertension and enhances contractility of vascular smooth muscle cells. Circulation 2008; 117 (16): 2114-22.

32. Itoh A, Nishihira J, Makita H, Miyamoto K, Yamaguchi E, Nishimura M. Effects of IL-1beta, TNF-alpha, and macrophage migration inhibitory factor on prostacyclin synthesis in rat pulmonary artery smooth muscle cells. Respirology 2003; 8 (4): 467-72. 